International Journal of Engineering \& Technology, $7(2.19)(2018) 12-16$
International Journal of Engineering \& Technology
SPC
Website: www.sciencepubco.com/index.php/IJET
Research paper

\title{
Multicast Utility Maximization with Load Balanc- ing in Private Cloud Environment
}

\author{
N Senthamarai ${ }^{1 *}$, M Vijayalakshmi ${ }^{2}$ \\ ${ }^{1}$ Assistant professor, CSE, Veltech Rangarajan Dr.Sagunthala R D Institute \\ of Science and Technology, Tamil Nadu \\ ${ }^{2}$ Assistant professor.Sr.grade, IST, Anna University, Tamil Nadu \\ *Email: senthamaraivijay@gmail.com
}

\begin{abstract}
Multicast is a technique for one-to-many communication over the network. It plays important role in cloud computing and reduces the transmission overhead in the private cloud environment. In this paper, build an efficient multicast tree for the multicast routing problem in which a network consists of different categories of nodes, where each category can have one or more nodes of the same characteristic which is different from the characteristics of nodes from other categories. So it is used to reduce the message traffic in such a network, to build a multicast tree and minimize the queuing delay using multicast selection algorithm.
\end{abstract}

Keywords: Cloud Computing; Multicast; Multicast Tree; private Cloud; Routing.

\section{Introduction}

As the distributed computing technologies are growing day by day, the need of communication and computation are rapidly increasing. The cloud computing is the suitable environment for efficient communication because a cloud environment consists of five unique characteristics that differentiate it from traditional computing. A private network consists of different categories of nodes, where each category can have one or more nodes of the same characteristic which is different from the other categories. So it is used to reduce the message traffic in such a private network, proposed a multicast tree that contains at least one node from each category by using the multicast selection algorithm for finding the shortest path computation to minimize the queuing delay.

A multicast packet is delivered to all the receivers belong to a group along a network structure such as tree or mesh; this is constructed from one multicast group. The owner of each computer on a private network would set aside a portion of its resources such as processing power, disk storage, or network bandwidth to be made directly available to other network nodes, without the need for central coordination.

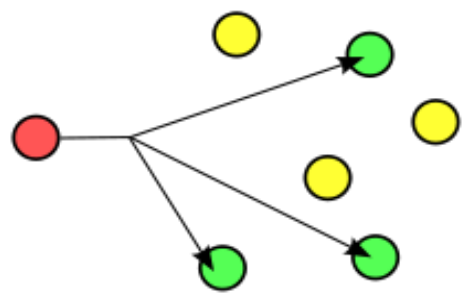

Fig.1: Multicast packet

In existing multicast protocols built in private cloud are based on the design of multicast on the wide area network. Before use the multicast concept in Private network, the proposed system need to carefully investigate whether these Internet-oriented multicast technologies can well for private cloud.

In this paper, we explore the multicast utility maximization and achieve the minimum queuing delay in private cloud.

\section{Literature Review}

The various multicasting methods reduce network traffic and improving different parameters in the cloud has been studied in different aspects.

In [1], proposed the optimizing the utilization of virtual resources in a cloud, including dynamic load balancing with virtualization. The Barr [2] mentioned the method of elastic load balancing inside of a virtual private cloud, but does not increase the throughput Chiba et al [3] proposed optimized load balanced algorithms for data transfer via multicast between an Amazon S3 server to Amazon EC2 instances. Their work focuses mainly on the optimized load on distribution of network and less on the problems of enabling multicasting with load balancing.

The Shu Fan, Honglin Zhao [4] was described about utility maximization in wireless networks. Their work focused on optimal value on network stability, but lead to poor delay performance.

In [5], proposed a randomized algorithm with performance guarantees. In [6], described single points of failure, imitated multicast transmissions can be used to distribute copies of critical data to various geographically disseminate locations on the grid area.

Authors X.Lin and N.B.Shroff was described about utility maximization for communication networks with multipath routing. This utility maximization problems appear naturally in several resource allocation problems in communication networks, such as the multi-path flow control problem, the optimal quality-of-service 
(QoS) and routing problem[7][8]. They have developed a lot of distributed algorithm for the utility maximization problem in communication networks that have the capability to allow multipath routing.

In [9][10], authors are provide a shortest path approach for distributed network utility maximization coding based on multicasting. The lagrangian relaxation method is applied to break down the issue into sub issues involving one destination each. Network coding techniques are used for least cost transmission scheme in multicast sessions. Network coding techniques improve network throughput, network robustness and the efficiency of network resource allocation over those achievable by pure routing.

Supratim Deb et al [11] present congestion control in networks which support both unicast and multicast sessions. A decentralized algorithm which enables the different rate-adaptive receivers in different multicast sessions to change their information rates to fulfill some reasonableness measure. In [12], Congestion controls achieves high bandwidth utilization over networks operating multi-path routing

In [13], MVNE model achieved a lower blocking ratio, higher revenue, and balanced network utilization. In [14], defined the multicast virtual network restoration problem and demonstrate its NP-complete nature in arbitrary graphs. Moreover, they demonstrated that the issue can be illuminated in polynomial-time in multi-established treelike server farm arrange topologies. In [15], the paper talked about the issue of how to boost the benefit of a private cloud in hybrid clouds while guaranteeing the service delay bound of delay-tolerant tasks. This paper proposed a profit maximization algorithm (PMA) to discover the temporal variation of prices in hybrid cloud. The temporal task scheduling provided by PMA can dynamically schedule all arrival tasks to execute both in private and public clouds. The sub problem of PMA is solved by the proposed hybrid heuristic optimization algorithm and simulated annealing particle swarm optimization (SAPSO). Besides, SAPSO is compared with existing baseline algorithms. It maximizes the throughput and the profit of a private cloud and also guaranteeing the service delay bound.

In [17], explained how to implement multicast support on AWS in a private cloud. However, this does not improve load balancing.

\section{Background and Design}

In this area, we talk about the foundation of private cloud multicast and outline.

\subsection{Private Cloud Model}

A private cloud is a protected deployment model of cloud computing that includes a distinct and secure cloud based environment in which only the predetermined client can operate. Similarly as with other cloud models, private clouds will provide computing power as a service within a virtualized environment using a hidden pool of physical computing resource. In any case, under the private cloud model, the cloud (the pool of resource) is only accessible by a single organization providing that organization with more prominent privacy and security. Private cloud administrations draw their asset from a particular pool of physical PCs however these might be facilitated inside or remotely and might be gotten to crosswise over private rented lines or secure encoded associations through open systems. For example, a private cloud service could be utilized by a financial company that is required to store sensitive data internally [18].

\subsection{Multicast Utilization}

The Multicast is the term used to depict correspondence where a snippet of data is sent from one or more points to a set of other points. For this situation there is might be at least one sender, and the data is disseminated to an arrangement of recipients. A multicast group is an arbitrary group of receivers that conveys an interest in receiving a particular data stream. This group has no physical or geographical limits the host can be located anywhere on the Internet or any private network. Hosts that are involved in receiving data flowing to a specific group must join the group using IGMP (Internet Group Management Protocol (IGMP).The other is Protocol Independent Multicast (PIM), which is used by routers to create multicast trees. When a router receives a request to join a stream via IGMP, it utilizes PIM to route the information stream to the proper framework [19].

\subsection{Path Computation and Selection}

The path calculation process to compute the best paths from all the other nodes to itself in the network and a node only has to advertise the data to its neighbors along with best path. Multicast routing fulfills the optimality prerequisite, which means a node can definitely recognize a widest path to every destination through advertisement from its neighbors. Each neighbor can distinguish its own particular best way.

\subsection{Queuing Delay}

The most extreme lining delay is corresponding to support measure. The more extended the line of parcels holding up to be transmitted, the more drawn out the normal holding up time is; and when the cradle fills the switch must drop bundles. At the point when the transmission convention utilizes the dropped-parcels side effect of filled supports to manage its transmit rate, as the Internet's TCP does, data transmission is genuinely shared at close theoretical limit with negligible system blockage delays.

\subsection{Multicast Transmission}

This innovation addresses packets to a group of recipient rather than the single recipient thus utilizing lesser bandwidth. Sending message to a single host like unicast or sending messages to all host like broadcast it select a group of host to form a distributed tree from source to receivers. Router creates distributed tree that create the path that IP multicast traffic to deliver traffic to all receivers in the network. Router will dynamically modify the distributed tree and maintain shortest path information from source to the group of receiver. Finally the packets are transmitted from source and reach the destination through this path.

\section{Multicast Selection Algorithm}

For utility expansion in single tree multicast situations, routers enable functionality. The multicast session rate over a connection on the tree is the most extreme of the rate of every downstream recipient of the session. A multicast selection algorithm is used for viewing the links over the LAN or WAN when the resolution or frame rate of the recorded data is too high for the available bandwidth.

\subsection{Multicast Tree Construction}

Amid the tree development, the server $\mathrm{S}$ dependably monitors which node in the existing tree has the greatest bottleneck bandwidth. Along these lines, each time another node joins the tree; it first contacts the server, which in turns reveals to it which node to pick as parent. In tree development, only the bottleneck bandwidths of the new node and its parent node are refreshed. These two nodes need to answer to the server. The aggregate messaging overhead of the tree development is $3 \mathrm{~N}$.

The tree development algorithm requires nodes to join the tree in the arranged order of their access link capacities. In any case, a client may join or leave the multicast session whenever. It is pro- 
foundly costly to dependably deteriorate the old tree and develop another one starting with no outside help when such an occasion happens.

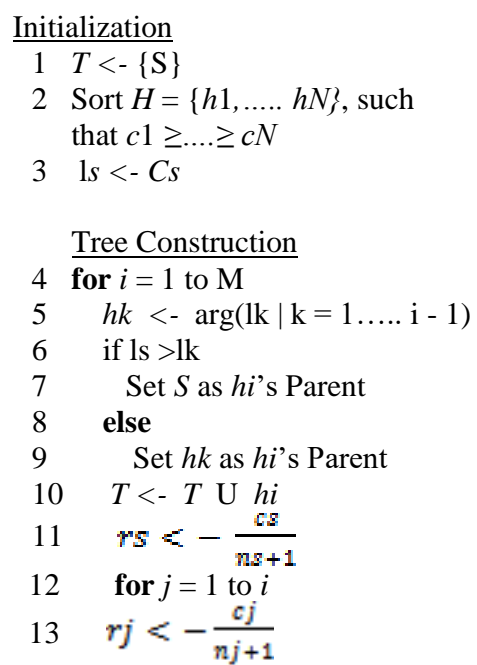

\subsection{Shortest Path Formation}

The processing path that takes the shortest time to complete of all parallel paths in a process instance, where each path considered begins at a starting node or an input to the process and ends at a terminate node. Fig.2. shows the tree structure form from root to leaf node.

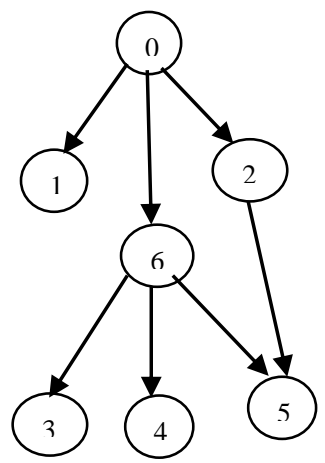

Fig.2: Tree structure Formation

The Probability of selecting path is given in equation,

$$
P(N)=\frac{1-D r}{D m}
$$

Where $\mathrm{P}(\mathrm{N})$ is the probability of selecting node. Dr is the dropping ratio of child and Dm is the delay between node children to parent.

\subsection{Multicast Selection Algorithm}

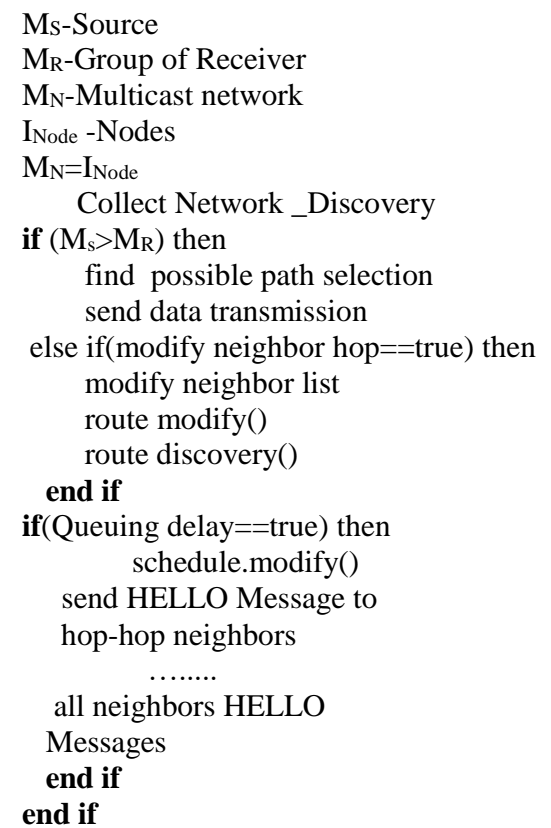

\section{Implementation and Analysis}

The proposed method of the multicast selection algorithm is implemented and analyzed in the cloud simulator. The performance is measured with respect to metrics like Packet throughput Ratio, Packet delivery ratio, end-end delay etc. When a network connection increases, more packets fail to reach the destinations due to packet collisions and channel contention caused by redundant retransmissions of packets. Therefore, the waiting time of data packets in the queue increases. It can still achieve comparable performance levels in terms of throughput when contrasted with the other schemes for various network connections.

Throughput $=\frac{\operatorname{Re} \text { ceivingPadets }}{\text { Time }}$

Fig. 3 shows the Data packet delivery ratio analysis. This is the proportion of aggregate number of packets successfully received by the destination nodes to the number of packets sent by the source nodes throughout the simulation.

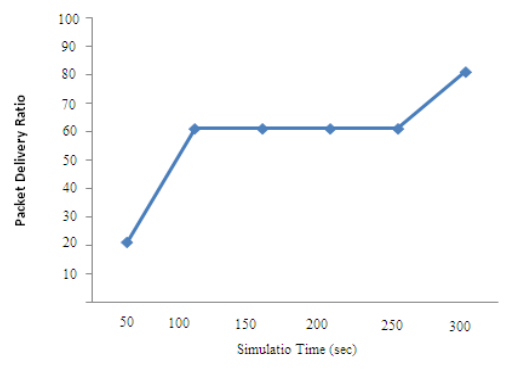

Fig.3: Data packet delivery ratio

The multicast transmission performance is analyzed and tested in cloud simulator. Each node hosted 50 hosts. 10 multicast groups is created, almost 20 hosts from different nodes have joined for the same multicast group. The following Table 1 and Figure 4 show the multicast transmission from one sender to one group of nodes. The packets are reached to the destination using multicast section algorithm and also reduces the delay. 
Table 1: Multicast Transmission

\begin{tabular}{|l|c|c|c|c|c|}
\hline Source IP & $\begin{array}{c}\text { Destina- } \\
\text { tion IP } \\
\text { Address }\end{array}$ & $\begin{array}{c}\text { dst } \\
\text { port }\end{array}$ & $\begin{array}{c}\text { Pack- } \\
\text { ets per } \\
\text { Sec } \\
\text { (pps) }\end{array}$ & $\begin{array}{c}\text { Avg } \\
\text { Bytes } \\
\text { per } \\
\text { Packet }\end{array}$ & $\begin{array}{c}\text { K bits } \\
\text { per sec } \\
\text { (kbps) }\end{array}$ \\
\hline 192.10 .1 .1 & 10.10 .1 .40 & 19996 & 94 & 720 & 640 \\
\hline 192.10 .1 .1 & 10.10 .1 .40 & 19998 & 94 & 732 & 658 \\
\hline 192.10 .1 .1 & 10.10 .1 .40 & 19940 & 98 & 782 & 760 \\
\hline 192.10 .1 .1 & 10.10 .1 .40 & 19938 & 72 & 644 & 585 \\
\hline 192.10 .1 .1 & 10.10 .1 .40 & 19923 & 88 & 778 & 780 \\
\hline 192.10 .1 .1 & 10.10 .1 .40 & 19935 & 70 & 610 & 530 \\
\hline 192.10 .1 .1 & 10.10 .1 .40 & 19957 & 72 & 632 & 576 \\
\hline 192.10 .1 .1 & 10.10 .1 .40 & 19943 & 86 & 784 & 792 \\
\hline
\end{tabular}

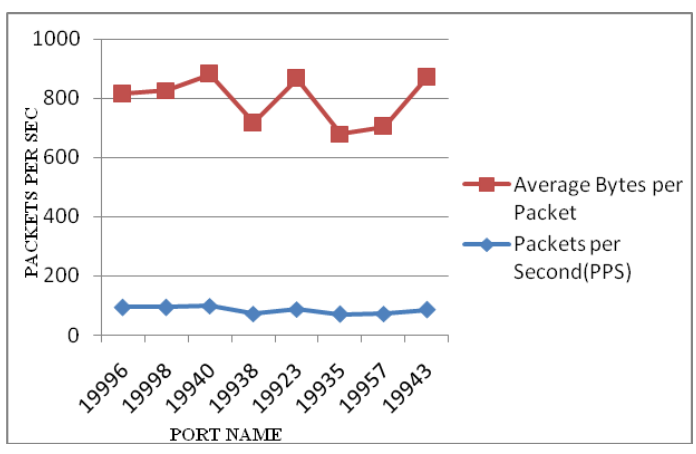

Fig.4:Host versus Delay

The following Table 2 and Fig.5 shows the multicasting transmission in switch. The Link speed is determined based on the size of the switch.

Table 2: Multicast in switch

\begin{tabular}{|l|c|c|c|c|}
\hline $\begin{array}{l}\text { Switch } \\
\text { Name }\end{array}$ & $\begin{array}{c}\text { Input } \\
\text { Packets }\end{array}$ & $\begin{array}{c}\text { In } \\
(\text { Pps })\end{array}$ & $\begin{array}{c}\text { Output } \\
\text { Packets }\end{array}$ & $\begin{array}{c}\text { Out } \\
(\text { Pps })\end{array}$ \\
\hline AAA & 2058834 & 10 & 7345862 & 19 \\
\hline BBB & 2509289 & 9 & 6740592 & 21 \\
\hline CCC & 8625688 & 90 & 10558315 & 20 \\
\hline DDD & 2374154 & 23 & 71494375 & 9 \\
\hline
\end{tabular}

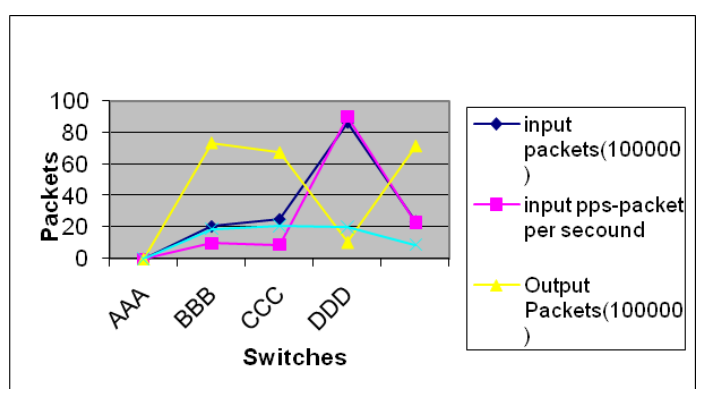

Fig.5: Packet Transmission

The comparison of Normal Multicast and Multicast Selection Algorithm is shown in the following Fig. 6.

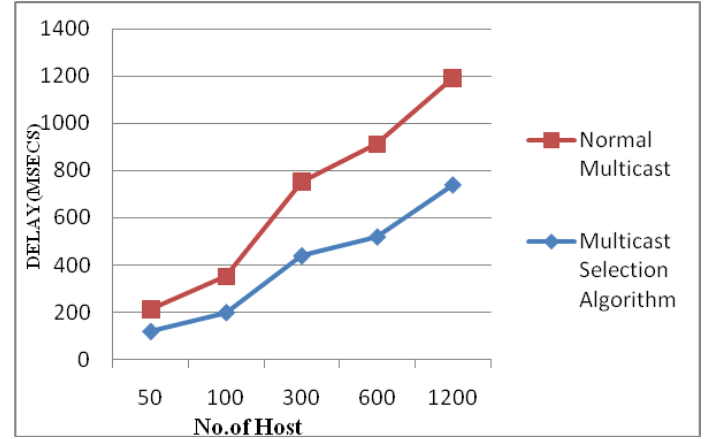

Fig. 6:Normal Multicast VS Multicast Selection Algorithm

\section{Conclusion and Future Work}

This Paper deals with the design, implementation and a detailed study of how the multicast tree constructed from the single sender to multi receiver. Multicast routing in cloud computing compared to content delivery networks does not require any dedicated infrastructure. It is self scaling because network usage increases according to the number of users. Multicast selection algorithm is used to identify the shortest path to minimize the delay during the multicast transmission. Through analysis and experiments can select the shortest path formation based on the multicast selection algorithm to transfer the packets in tree formation. In future, the multicast transmissions are performed for eliminating the packet loss during transmission.

\section{Acknowledgement}

I would like to thank my family, friends and colleagues, for their continuous motivation and support.

\section{References}

[1] Amit S. Rodge, Chandan Pramanik, Joy Bose, Sandeep Kumar Soni,"Multicast Routing with Load Balancing Using Amazon Web Service" 978-1-4799-5364- 6/14,IEEE, 2014

[2] Dylan A.P. Davis, Jeremy M. Plante, and Vinod M. Vokkarane, "Critical Resource Multicast Protection in Data Center Networks",978-1-4673-6432-4/15, IEEE ICC 2015- Next Generation Networking Symposium.

[3] Deb S. and Srikant R. "Congestion control for fair resource allocation in networks with multicast flows". IEEE/ACM Trans. Network, vol. 12 , no. 2, pp. 274-285, 2004

[4] Haitao Yuan, Jing Bi, Wei Tan, and Bo Hu Li," TemporalTask Scheduling With Constrained Service Delay for Profit Maximization in Hybrid Clouds", 1545-5955 (C) 2016 , IEEE Transactions on Automation Science and Engineering.

[5] Jeff Barr, "AWS Elastic Load Balancing Inside of a Virtual Private Cloud", Amazon Web Services Blog, 2011. URL:http://aws.typepad.com.

[6] Lin X.and Shroff N. B "Utility maximization for communica tion networks with multipath routing", IEEE Trans. Autom. Control vol. 51 no. 5, pp. 766-781, 2006.

[7] Lijun Chen, Tracey Ho, Mung Chiang, Steven H. Low, and John C.Doyle, "Congestion Control for Multicast Flows With Network Coding," IEEE Trans. on information theory, vol. 58, no. 9, 2012.

[8] M.Bencek, S.Buckell, S.Struk", How to enable broadcast and multicast support on Amazon (AWS) EC2".

URL: https://www.buckhill.co.uk/blog/how-to-enable-broadcast-and multicast-on-amazon-aws-ec $2 / 2$

[9] Naixue Xiong, Xiaohua Jia, Laurence T. Yang, Athanasios V.Vasilakos, Yingshu Li, and Yi Pan, "A Distributed Ef ficient Flow Control Scheme for Multirate Multicast Neworks," IEEE Trans. on Parallel and distributed systems, vol.21, no..9, 2010.

[10] Shu Fan, Honglin Zhao, "Modular Control for Throughput Utility Maximization in Multi hop Wireless Networks", 9781-5090-1698-3/16@2016 IEEE. 
[11] Sara Ayoubi, Chadi Assi, Lata Narayanan, and Khaled Shban MINTED: Multicast VIrtual Network Embedding in Cloud Data Centers with Delay Constraints", IEEE Transactions on communications, vol. 63, no. 4, 2015.

[12] T. Chiba, M. den Burger, T.Kielmann, S. Matsuoka, "Dynamic Load-Balanced Multicast for Data- Intensive Applications on Clouds", 10th IEEE/ACM International Conference on Cluster,Cloud and Grid Computing, 2010.

[13] Yingjie Bi, Chee Wei Tan and Ao Tang," Network Utility Maximization with Path Cardinality Constraints", IEEE Infocom 2016 - The 35th Annual IEEE International Conference on Computer Communications.

[14] Vinh Bui, Weiping Zhu, Alessio Botta, "A Markovian Approach to Multipath Data Transfer in Overlay Networks," IEEE Trans on parallel and distributed systems,Vol.21,No.10, 2010.

[15] Wu Y. and Kung S. Y "Distributed utility maximization for network Coding based multicasting: A shortest path approach," IEEE J. Sel. Areas Commun. vol. 24, no. 8, pp.14751488, 2006.

[16] Sara Ayoubi, Chadi Assi Lata Narayanan, and Khaled Shaban," Optimal Polynomial Time Algorithm for Restoring Multicast Cloud Services" IEEE Communications Letters, vol. 20, no. 8, 2016.

http://www.interoute.com/cloud- article/what-private-cloud http://techterms.com/definition/multiCasting 